EPJ Web of Conferences 53, 08003 (2013)

DOI: $10.1051 /$ epjconf/20135308003

(C) Owned by the authors, published by EDP Sciences, 2013

\title{
Reconstruction of the muon production depth with ground array data based on the TTC (Time-Track Complementarity) approach
}

\author{
M. Ambrosio ${ }^{1}$, C. Aramo ${ }^{1}$, M. Cilmo ${ }^{1}$, R. Colalillo², F. Guarino ${ }^{2}$ and L. Valore ${ }^{2}$ \\ 1 INFN Sezione di Napoli, via Cintia 2, 80125 Napoli, Italy \\ 2 INFN Sezione di Napoli and Dipartimento di Fisica, Universita' "Federico II", via Cintia 2, \\ 80125 Napoli, Italy
}

\begin{abstract}
The muon longitudinal profile along the shower axis depends on the nature of the primary particle and primary hadronic interaction with air nuclei. The measurement of muonic component inside showers generated by Very High Energy Cosmic Rays provides a very powerful tool for sensing high energy interactions between cosmic ray particles and air molecules. Fundamental parameters such as the interaction cross section, inelasticity, hadron production and multiplicity can be measured by comparing the development of shower electromagnetic component with that of muonic component. Since 1992 a method has been developed to combine the muon arrival direction in a ground based array for cosmic ray detection with their arrival delay with respect to the shower core. This combination permits to select high energy muons weakly scattered in the atmosphere and to reconstruct their height of production with good accuracy. In this paper we discuss the possibility to realize a "dual" apparatus able to detect both electromagnetic and muonic component at primary energies greater than $10^{17} \mathrm{eV}$.
\end{abstract}

\section{INTRODUCTION}

There are few Ultra High Energy Cosmic Rays (UHECR) experiments operating in the energy range above $10^{17} \mathrm{eV}$, where the very low flux of primary particles requires the coverage of a very large area in which detectors must be scattered. At an energy greater than $10^{17.5} \mathrm{eV}$, two detecting techniques can be used. The first one is the traditional technique using a ground based array of particle detectors whose area and spacing depend on the energy range to be covered. In the case of the Pierre Auger Observatory a grid of 1660 water Cerenkov detectors $1.5 \mathrm{~km}$ apart, instruments an area of $3.000 \mathrm{~km}^{2}$. The array permits observation of shower front particles (e, $\gamma, \mu$ and hadrons) and reconstruction of shower axis and energy. The second technique is based on the use of fluorescence, the light generated along the shower axis from particles interacting with atmospheric nitrogen. This observation permits reconstruction of the development of the electromagnetic component of the shower in the atmosphere, closely connected to the primary particle nature and nuclear cross sections.

Unfortunately both techniques cannot permit a precise estimation of primary nature and hadronair cross section because there are no robust theoretical models available for energies beyond the accelerator limits. In addition, CR interactions with air nuclei develop along the shower axis in the forward region, not fully explored by accelerators. At the moment, cross section measurements of UHECR with air nuclei performed by various experiments suffer from large errors, introduced by the different experimental techniques and interaction model uncertainties.

For what concerns the cosmic ray $(\mathrm{CR})$ primary mass estimation, the main confusion is generated by the apparent transition from heavy to light nuclei observed at energies greater than $10^{18} \mathrm{eV}$. This

This is an Open Access article distributed under the terms of the Creative Commons Attribution License 2.0, which permits unrestricted use, distribution, and reproduction in any medium, provided the original work is properly cited. 


\section{EPJ Web of Conferences}
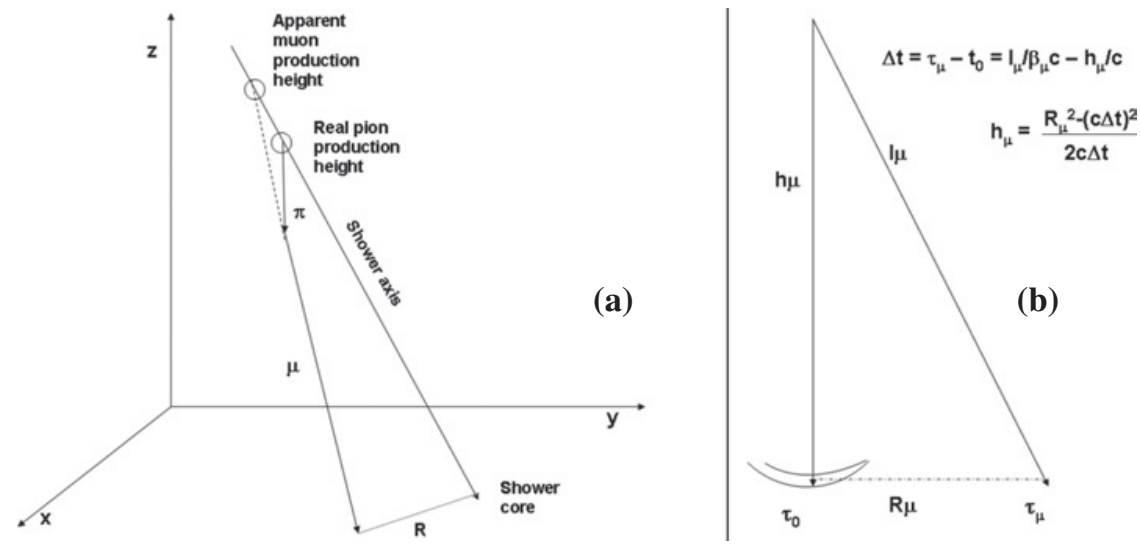

Figure 1. a) Apparent height of production for muons generated by pion decay as reconstructed by tracking; b) Apparent height of production for muons generated by pion decay as reconstructed by muon arrival time.

appears also as an excess of muons in the showers at these energies, not reproduced by the Monte Carlo simulations based on the known cross section values. So, the open problems for UHECR physics consists on how to obtain precise measurements on the primary particle nature, how to measure CR-air cross sections, and how to estimate parameters of hadronic interactions occurring in the first interactions (inelasticity, pion multiplicity, pseudorapidity, etc.).

In 1995 John Linsley [1, 2] proposed a new approach to face this problem. He suggested detection of muons inside the shower front and the reconstruction of their heights of production along the shower axis. Muons are generated by the decay of pions produced in the hadronic interaction CRair and carry information to the ground about this process. Reconstruction of the muon production profile along the shower axis contains information about the hard processes involved and on interaction parameters. High energy muons generated in the first interactions can be selected by reconstruction of their height of production permitting the study of primary interaction and primary mass. In addition the difference in the lateral distribution between electromagnetic and muonic components at ground level is strictly correlated to the primary mass, as well as comparison between their elongation depths. So muons can offer a powerful tool for the UHECR physics. Unfortunately up to now the difficulty of discriminating muon from electronic components prevented this method to be successfully applied. Only partial information has been obtained from dedicated apparatus at the knee energy region, where KASCADE experiment used muons for primary mass discrimination confirming disagreements with Monte Carlo simulations based on known cross sections and interaction parameters [3].

In the following we describe the TTC (Time - Track Complementarity) approach suggested by Linsley and its application in previous experiments operating in the knee energy region. Then we discuss difficulties and possible solutions for applying the method at the UHECR energies. Finally we suggest a possible experimental program for the Pierre Auger Observatory.

\section{THE TTC PRINCIPLE}

Muons in extensive cosmic ray showers are generated by the decay of pions and kaons produced in hadronic interactions of the leading particle with air nuclei. They carry at ground level useful information on the interaction processes. Reconstruction of their height of production provides information on hadronic component development inside the shower. They do not originate along the shower axis, as reported in Fig. 1a, as a consequence the production height reconstruction can be affected by large errors, mainly in the proximity of shower core [4]. Muons heights of production can be also 


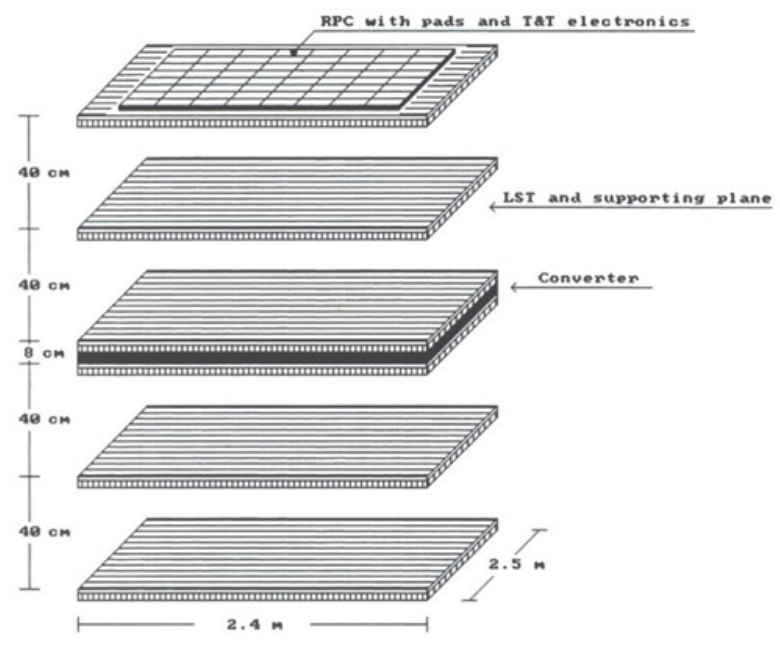

(a)

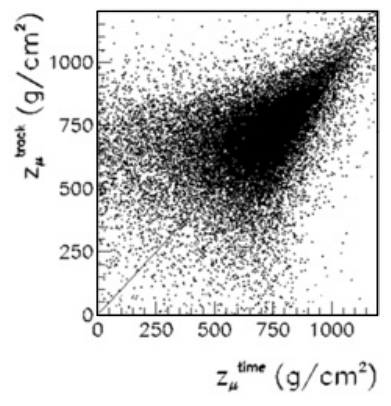

(b)

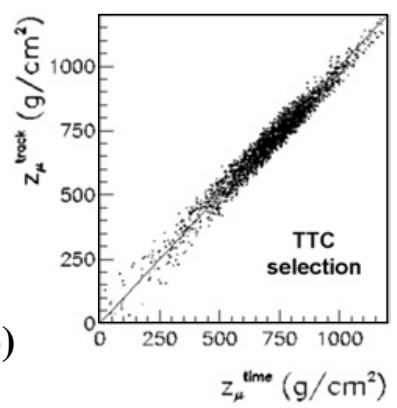

Figure 2. a) The G/C_P streamer tube telescope covered with a layer of four $\mathrm{m}^{2} \mathrm{RPC}$ equipped with $12 \times 12 \mathrm{~cm}^{2}$ pads; b) scatter plot ztrack vs ztime for all events (upper) and events selected with TTC selection criteria (lower).

reconstructed measuring their arrival time at the observation level respect to the arrival time of shower core [5]. In this case we must suppose that the muon originates along the shower axis and travels directly the atmosphere with relativistic velocity (Fig. 1b). Obviously both methods present significant errors. Travelling in the atmosphere muon are deviated by the Earth's magnetic field and scattered by the air nuclei. This effect becomes more important for showers at large zenith angles, since the muon path in the magnetc field becomes larger. Experimental errors in the measurements of the axis inclination and arrival time of the shower core play a fundamental role in the accuracy of reconstruction.

The Time-Track Complementarity approach is based on the assumption that muons whose production heights reconstructed by tracking are coherent with those from timing are the most energetic and less deviated by the Earth magnetic field and by multiple scattering. Then their production height measurement is more accurate and permits one to identify the muon production along the shower axis. Results of Corsika Monte Carlo simulations at the knee energy region have been performed by several authors [4-6] and some experimental measurements have been attempted in the past.

\section{PREVIOUS EXPERIMENTS USING TTC}

The TTC approach requires muon detection in a ground based array and measurement of their arrival time and direction. At the knee energy region only two experiments attempted to face the problem using Resistive Plate Counters (RPC) as muon detector for arrival time measurements and streamer tube telescopes for arrival direction identification. The first one, GREX_COVER PLASTEX (G/C_P), was built inside the GREX array in Haverah Park [7]. Four streamer tube telescopes made of six layers divided by a lead layer (Fig. 2a) were covered with $4 \mathrm{~m}^{2}$ RPC layers equipped with $12 \times 12 \mathrm{~cm}^{2}$ pads. Muons identified and tracked by means of streamer tube telescopes were detected also by RPC measuring their arrival time [8].

The experiment was able to measure for the first time the thickness in time of shower front at the knee region $[9,10]$. Nevertheless it was not able to reconstruct the muon height of production 


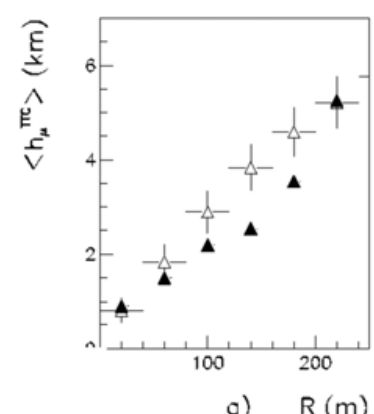

o) $\quad R(m)$

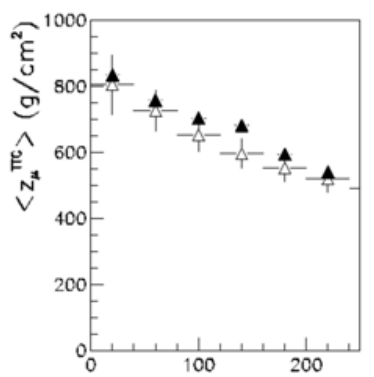

b) $R(m)$

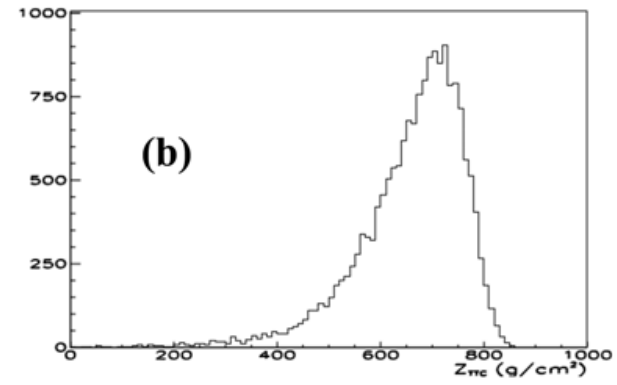

Figure 3. a) Comparison between the muon height of production (h) and slant production depth (z) obtained from simulations $(\Delta)$ and experimental data $(\boldsymbol{\Delta})$; b) integrated muon production slant depth.

because of the impossibility to correlate the arrival time of muons with the arrival time of shower core at ground level.

The second attempt was the installation of a $40 \mathrm{~m}^{2}$ RPC layer below the EAS_TOP calorimeter at the top of the Gran Sasso Mountain. A $12 \times 12 \mathrm{~cm}^{2}$ calorimeter made of 9 streamer tube layers separated by 8 concrete layers was used to select and track muons with energy greater than $2 \mathrm{GeV}$. The calorimeter was built inside the EAS_TOP array at an altitude of $2100 \mathrm{~m}$. The RPC layer measured the arrival time of muons crossing the calorimeter, allowing correlation between track and time. Figure $2 \mathrm{~b}$ shows the muon production slant depth along the shower axis reconstructed using tracking and timing separately and imposing TTC correlation. Result is impressive: Fig. 3a shows the comparison between Corsika Monte Carlo simulated and measured muon height and the slant depth of production. Figure $3 \mathrm{~b}$ reports the muon longitudinal development as measured in this experiment. At the moment this measure is the only one obtained in the knee energy region $[11,12]$.

\section{POSSIBLE APPLICATIONS TO UHECR APPARATUS}

The TTC technique can be applied to giant arrays at energies greater than those in the knee energy region, where complementary information coming from detection of electromagnetic components at ground level and from fluorescence radiation detection emitted along the shower axis provide information on particle distributions at the observation level and on leading particle interactions along the shower path in the atmosphere. From this point of view, the Auger apparatus is ideal for its complexity, its infill array and, maybe, for the possibility to separate the muon signal in the Cerenkov ground detectors from the electromagnetic one, providing complementary measurement of muon density in the entire observation field [13]. In the following we illustrate a possible improvement of Auger infill subarray to make it able to detect muons providing tracking and timing information for reconstruction of muon production height. While the Auger array has a grid of $1.500 \mathrm{~m}$ between Cerenkov detectors, in the infill the grid has been reduced by half (Fig. 4a), lowering the shower threshold to $10^{17} \mathrm{eV}$. Fluorescence detectors covering the infill have been complemented by High Elevation Telescopes (HEAT) to detect nearby showers [14]. Various groups have installed muon detectors in this subarray using it as test array for muon detection (AMIGA, RPC, BATATA) and for testing new techniques. Then the field is ideal for eventual experimentations of new approaches.

Figure 4a shows a possible evolution of the Auger infill subarray including seven RPC (Resistive Plate Detector) telescopes for tracking and timing of muons spaced according a $1.500 \mathrm{~m}$ grid. RPCs are well known large area, fast and cheap, detectors used in a lot of experiments to provide muon detection. They can be equipped with $3 \mathrm{~cm}$ strips for tracking and $12 \times 12 \mathrm{~cm}^{2}$ pads for timing giving an accuracy of fraction of milliradiant for tracking and nanosecond for timing. To select muons inside the shower 

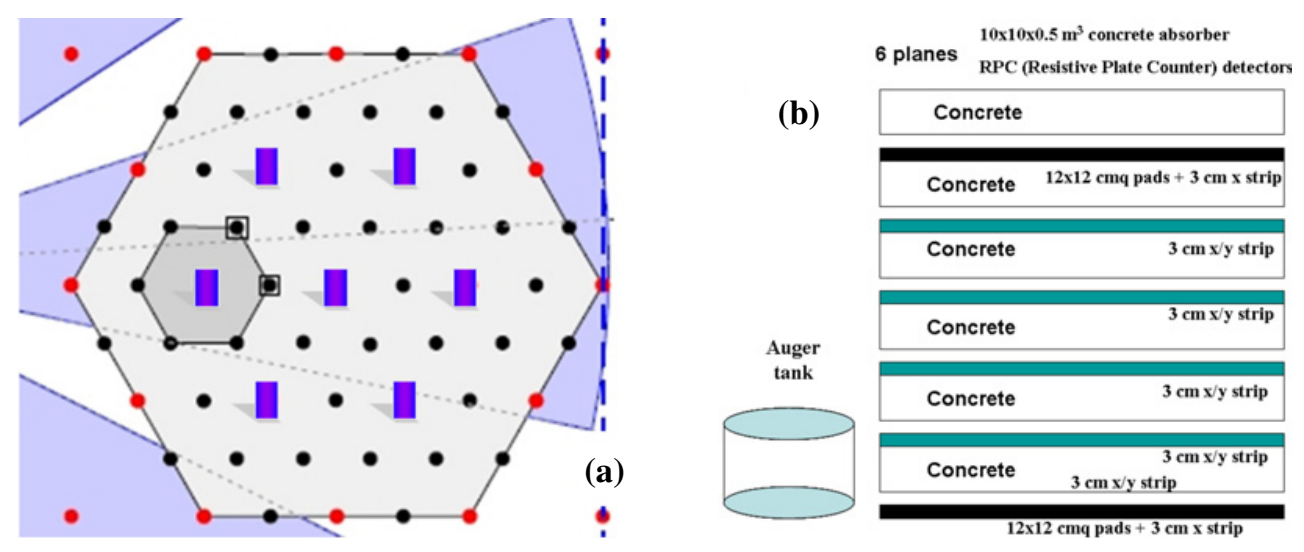

Figure 4. a) Possible layout of the Auger infill subarray with indication of muon telescopes position; b) a possible layout of muon telescope suggested.
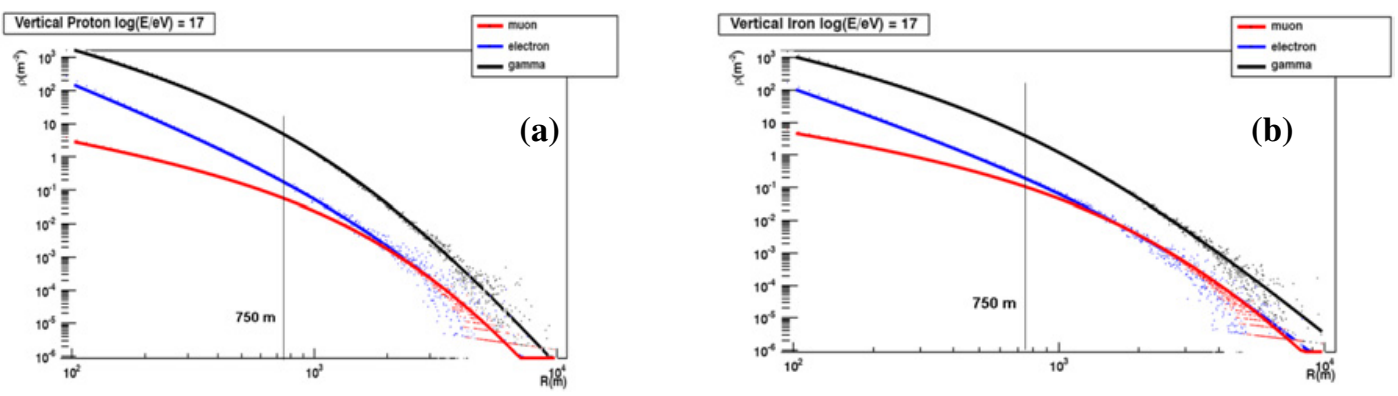

Figure 5. Particle density at the observation level for vertical $10^{17} \mathrm{eV}$ proton (a) and iron (b) nuclei.

front, a thick absorber is needed. Figure $4 \mathrm{~b}$ shows a possible layout for telescopes of $100 \mathrm{~m}^{2}$ each: 6 concrete planes mask 6 RPC planes absorbing electrons, gammas and hadrons and imposing to muons a $2 \mathrm{GeV}$ energy threshold. The concrete planes are necessary to discriminate muons from other particles inside the shower front.

With this configuration, the maximum core distance from nearest telescope is $750 \mathrm{~m}$. As shown in Fig. 5a and $5 \mathrm{~b}$, at that distance for $10^{17} \mathrm{eV}$ showers the density of muons originating from protons are about $0.1 \mu / \mathrm{m}^{2}$ while density of muons originating from iron primary particles is three times larger. This means that several tens of muons can be detected for each event from at least three telescopes with good tracking and timing accuracy. At higher energy, or closer to the core, hundreds of muons can be observed allowing muon development reconstruction along the shower axis for individual event. The resulting information is very powerful: each shower can be observed, separating its content of electromagnetic components from muonic one. Comparison of relative density at ground level and of shower development in the Earth's atmosphere allows a powerful tool for understanding the hadronic interactions occurring and for separating the contribution of proton and iron primary nuclei. The complementary information on muon density at ground level can be obtained by using additional shielded detectors inside the array [15] or using the Auger Cherenkov tanks if the separation of signal generated from muons can be disjoined from the global signal in the detector [16] or applying the universality principle [17, 18].

Such "dual" apparatus inside the Auger infill array would allow to detect muons and reconstruct the full shower characteristics with unprecedented accuracy. This would allow for the first time a direct 
comparison of the proton-proton cross section and hadronic interaction properties measured in cosmic rays experiments and accelerators at the same energy. This measurement would allow the development and fine tuning of new Monte Carlo programs and, more importantly, would validate muon detection strategies to be adopted at higher energies on the full Auger array.

\section{CONCLUSION}

The TTC approach works well in the knee energy region as demonstrated by the EAS_TOP experiment. If applied to a giant array such as the Pierre Auger Observatory it would permit to estimate the muon production depth and compare the muon longitudinal development with that of electromagnetic component depicted with fluorescence detectors. In addition the muon content at ground level can provide a measurement of muon Lateral Distribution Function (LDF) and muon density. In this case the comparison between muon and electromagnetic information will provide a powerful tool to estimate the primary mass and hadronic cross section at energies higher than that of actual accelerators. New physics can be at the origin of the observed discrepancies between Monte Carlo simulations and primary composition measurements and a new horizon can be opened by a "dual" giant array able to reconstruct both the electromagnetic and muonic components on the same detected shower.

\section{References}

[1] J. Linsley, Il Nuovo Cimento 15C, N. 5 (1992)

[2] J. Linsley, 24th ICRC 1, 356 (1995)

[3] Kascade-Grande Collaboration, Astroparticle Physics 34, 476-48 (2011)

[4] T. V. Danilova et al., J. Phys. G: Nucl. Part. Phys. 20, 961 (1994)

[5] D. Dumora et al., J. Phys. G: Nucl. Part. Phys. 22, 273 (1996)

[6] M. Ambrosio et al. J. Phys. G: Nucl. Part. Phys. 23, 219 (1997)

[7] G. Agnetta et al., Nucl. Instrum. Meth. A 359, 596-602 (1995)

[8] G. Agnetta et al., Nucl. Instrum. Meth. A 381, 64-72 (1996)

[9] M. Ambrosio et al., Astropart. Phys. 7, 329-341 (1997)

[10] M. Ambrosio et al., Astropart. Phys. 11, 437-450 (1999)

[11] M. Ambrosio et al., Nucl. Phys. Proc. Suppl. 75, 315-317 (1999)

[12] A. Ambrosio et al., 26th ICRC 5, 312 (1999)

[13] D. Garcia-Gamez for the Pierre Auger Collaboration, 32th ICRC, HE1.3 0735 (2011)

[14] M. Ambrosio et al., NIM A 660 91-103 (2011)

[15] The Pierre Auger collaboration, arXiv:1107.4807v1

[16] A. Letessier-Selvon for the Pierre Auger Collaboration, these proceedings

[17] A. Yuskov et al., Phys. Rev. D 81, 123004 (2010)

[18] A. Yuskov for the Pierre Auger Collaboration, these proceedings 